Mots. Les langages du politique

$78 \mid 2005$

Usages politiques du genre

\title{
La féminisation des noms de métiers et des titres dans la presse française (1988-2001)
}

Itsuko Fujimura

\section{(2) OpenEdition \\ Journals}

Édition électronique

URL : https://journals.openedition.org/mots/355

DOI : $10.4000 /$ mots.355

ISSN : 1960-6001

Éditeur

ENS Éditions

Édition imprimée

Date de publication : 1 juillet 2005

Pagination : $37-52$

ISBN : 2-84788-080-1

ISSN : 0243-6450

Référence électronique

Itsuko Fujimura, "La féminisation des noms de métiers et des titres dans la presse française (1988-2001) », Mots. Les langages du politique [En ligne], 78 | 2005, mis en ligne le 31 janvier 2008, consulté le 23 avril 2022. URL : http://journals.openedition.org/mots/355 ; DOI : https://doi.org/ $10.4000 /$ mots.355

\section{(C) ENS Éditions}




\section{La féminisation des noms de métiers et des titres dans la presse française (1988-2001) ${ }^{1}$}

En France, la langue connait actuellement un grand bouleversement linguistique : la féminisation des noms de métiers et de titres. Ce processus, à la fois linguistique et politique, n'est pas une nouveauté en français. On avait déjà commencé à l'envisager depuis les années soixante-dix au Québec : elle faisait partie des réformes linguistiques qui devaient contribuer à la réalisation de l'égalité entre les hommes et les femmes dans la société. En France, cette question est devenue un sujet de débat public pour la première fois en 1983, durant le premier septennat de François Mitterrand ${ }^{2}$. La discussion a pourtant été close avec le changement de gouvernement en 1986, une fois publiée - mais jamais appliquée - la circulaire du Premier ministre qui avait déterminé les grandes lignes de la féminisation de la langue. Or, une dizaine d'années plus tard, en 1997, ce problème a été remis à l'ordre du jour sous le gouvernement Jospin.

Notre étude a deux objectifs. Nous présenterons tout d'abord la chronologie de cette féminisation en cours, en observant les usages dans la presse écrite française entre janvier 1988 et décembre 2001. Nous mettrons ensuite en évidence des facteurs qui conditionnent cette évolution de la langue, à savoir des facteurs sémantiques, lexicaux et sociolinguistiques. Puis nous examinerons la raison pour laquelle les noms concernant la politique, l'administration et la justice, comme ministre, député et juge, ont connu un changement immédiat dès 1998, tandis que ceux qui appartiennent aux domaines de la recherche ou des lettres comme professeur, chercheur, écrivain, se féminisent moins facilement.

\section{Corpus et méthode}

Pour mener à bien cette étude, nous nous sommes appuyée sur l'analyse des variations du genre grammatical des noms de métiers dont les référents sont des femmes. Dans ce but, nous avons constitué une banque de données

1. Je remercie Dominique Desmarchelier, Claire Dodane, Pierre Fiala, Alexandre Gras, Catherine Kerbrat-Orecchioni, Nakao Hiroshi, Okubo Tomonori, Juliette Rennes et les collègues du Nagoya University Corpus Project, qui m’ont aidée à mener à bien cette étude.

2. Les textes à retenir sont, entre autres, la circulaire du 11 mars 1986 relative à la féminisation des noms de métier, fonction, grade ou titre (Journal officiel du 16 mars 1986) et la Déclaration de l’Académie française du 14 juin 1984, par G. Dumézil et Cl. Lévi-Strauss.

Université de Nagoya, Faculté du développement international, fujimura@nagoya-u.jp 
d'environ 57 millions de mots à partir de périodiques français numérisés, dont Le Monde (1988, 1994, 1997, 1998, 2000 et 2001), Libération (1995 et 1999), Le Télégramme (1996 et 2000), Dernières Nouvelles d'Alsace (1996 et 2000, désormais DNA) et Le Point (1996 et 2000)33.

En premier lieu, nous avons établi une liste composée de 200 prénoms féminins fréquemment utilisés. Nous avons ensuite recherché automatiquement, dans la banque de données des périodiques, la cooccurrence des prénoms faisant partie de cette liste et d'une centaine de noms de métiers problématiques placés à moins de dix mots de chaque prénom. Enfin, nous y avons apporté une correction manuelle pour n'obtenir que des exemples utilisés pour définir les prénoms. Ainsi, nous avons pu obtenir 7000 exemples de noms de métiers et de titres dont les référents sont exclusivement féminins. La distribution est la suivante:

\begin{tabular}{|l|l|l|}
\hline Corpus & Année & Nb d'occurrences \\
\hline \multirow{4}{*}{ DNA } & 1996 & 256 \\
& 2000 & 660 \\
\hline total & & 916 \\
\hline \multirow{4}{*}{ Le Monde } & 1988 & 148 \\
\cline { 2 - 3 } & 1994 & 290 \\
\cline { 2 - 3 } & 1997 & 475 \\
\cline { 2 - 3 } & 1998 & 675 \\
\cline { 2 - 3 } & 2000 & 606 \\
\cline { 2 - 3 } & 2001 & 502 \\
\hline \multicolumn{2}{|l|}{ total } & 2696 \\
\hline \multirow{2}{*}{ Le Point } & 1996 & 328 \\
\cline { 2 - 3 } & 2000 & 446 \\
\hline total & 1995 & 774 \\
\hline \multirow{2}{*}{ Libération } & 1999 & 338 \\
\hline \multicolumn{2}{|l|}{ total } & 638 \\
\hline \multirow{2}{*}{ Télégramme } & 1996 & 574 \\
\cline { 2 - 3 } & 2000 & 1438 \\
\hline \multicolumn{2}{|l|}{ total } & 2012 \\
\hline \multicolumn{2}{|l|}{ TOTAL } & 7036 \\
\hline
\end{tabular}

Tableau 1: Données

L'ensemble des résultats qui suivent est statistiquement significatif avec le test du chi-carré au seuil de 0,05, ce qui signifie que les distributions observées diffèrent sensiblement de ce qu'aurait donné le hasard avec une marge

3. Voir détail des corpus en annexe. 
d'erreur inférieure à $5 \%$. Nous éviterons de mentionner systématiquement les chiffres afin d'alléger le fil de notre analyse.

\section{Chronologie de la féminisation}

La figure 1 montre clairement que le genre grammatical de ministre, dont les référents sont féminins, a subi un changement brusque en 19984. Dans tous les périodiques, ministre au féminin, qui n'existait guère avant 1998, est tout d'un coup apparu cette année-là en nombre et est désormais en usage ${ }^{5}$.

Figure 1: ministre

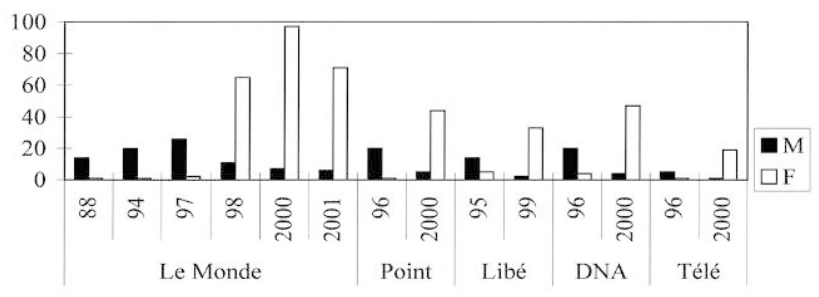

Il en va de même pour député(e). Ce mot présente un changement semblable ou même plus radical, comme l'indique la figure 2. L'usage de députée, très rare avant 1998, a brutalement augmenté après 1998 dans tous les périodiques, sauf dans Libération où le féminin était déjà commun en 1995. Nous pouvons signaler, comme exemple typique, l'inversion totale entre les deux genres qui s'est réalisé en quatre ans dans DNA.

Figure 2 : député(e)

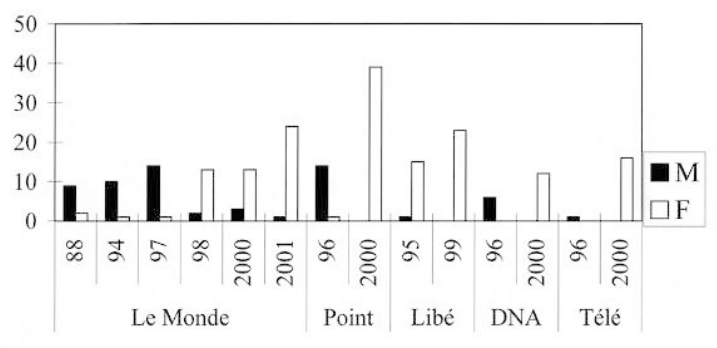

4. Dans tous les graphiques, les colonnes noires indiquent des occurrences de noms au masculin, les colonnes blanches des occurrences de noms au féminin. Le genre du nom a été déduit de l'accord grammatical, à savoir la forme du déterminant ou de l'adjectif et la forme du nom lui-même.

5. Voici un exemple de ministre au masculin, dans lequel l'italique désigne le nom de métier en question et les signes dièse le prénom qui lui est lié: «Le ministre de l'Intérieur néerlandais, Catarina \#lsabelle\# Dales, est décédée soudainement dans la nuit du dimanche 9 au lundi 10 janvier à son domicile d'Utrecht, à l'âge de soixante-deux ans » (Le Monde, 1994). 
Il va sans dire que ce phénomène s'explique surtout par l'effet de la circulaire du Premier ministre Lionel Jospin, publiée en mars 1998, au milieu d'une série de grands débats sur la langue et la place sociale des femmes amorcée dès la formation de son gouvernement en 1997. Cette décision a eu des conséquences certaines dans le secteur privé, notamment dans la presse, bien que la circulaire n'ait visé à proprement parler que la féminisation des noms dans les documents officiels.

Le changement ne s'est cependant pas produit au même rythme pour tous les noms. L'évolution de professeur est par exemple beaucoup plus lente que celle de ministre et de député(e), ainsi que l'indique la figure $3^{6}$. On y relève très peu de professeur au féminin, même après 19987, bien que la forme féminine de professeur soit proposée dans le guide d'aide à la féminisation des noms de métiers (Femme, j'écris ton nom..., publié en 1999 par la Documentation française), qu'elle soit effectivement utilisée dans les textes officiels, concernant par exemple l'Ordre de la Légion d'honneur (Journal officiel $n^{\circ} 97$, 23 avril 2000), et que le Nouveau Petit Robert paru en 2001 inscrive le mot professeur comme épicène au même titre que ministre. On ne voit cette tendance que dans Libération, qui tente d'éviter la forme visiblement masculine lorsqu'il est fait référence à une femme (on n'observe aucune occurrence en 1999) et de commencer à employer professeur au féminin ${ }^{8}$.

Figure 3 : professeur(e)

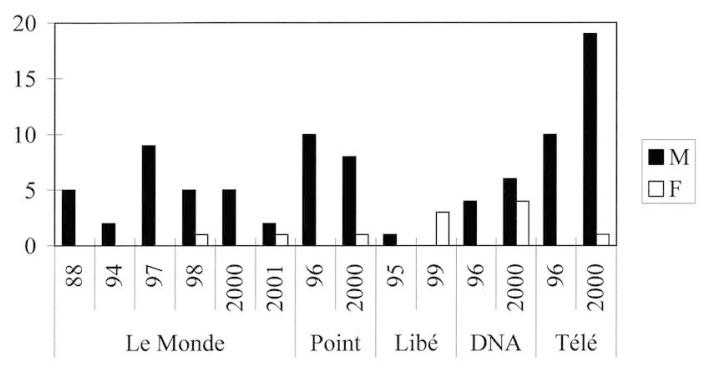

6. Cette figure présente des occurrences de professeur avec un déterminant et/ou un adjectif qui sont manifestement masculins ou féminins, ainsi que des occurrences de professeure, qui est indubitablement féminin. Les occurrences de professeur seul, sans aucune marque spécifique de genre, n'y sont pas prises en compte.

7. La féminisation est en cours. Dans un numéro plus récent du Monde (11 décembre 2002), on ne rencontre plus de professeur manifestement masculin mais deux cas de professeur féminisé: \#Nicole\# Benssoussan, professeure à l'université de Reims/la professeur \#Françoise\# Mignon.

8. Voici tous les professeur (e) au féminin relevés dans nos corpus : «professeur agrégée » (4 cas, DNA, 2000 ; Libération, 1999); " ancienne professeur de lettres » (Libération, 1999); «La professeur, \#Sylvie\# Cavaillès, repousse Jonathan » (Libération, 1999); «cette professeur de biologie» (Le Point, 200o); "cette ancienne professeur de lettres en LEP» (Le Monde, 1998); «professeur certifiée» (Le Monde, 2000); "sa professeur de bridge» (DNA, 2000); "son ancienne professeure de français» (Le Monde, 2001)/» une professeur de musique» (Le Télégramme, 2000). 
On attribue souvent les variations de rythme dans la féminisation aux difficultés morphologiques ou homonymiques rencontrées 9 . On dit par exemple que médecin ne se féminise pas parce que son correspondant féminin serait médecine, qui existe déjà avec un autre sens. Quant à professeur, on fait remarquer que ce nom n'est pas dérivé du verbe professer, mais directement du latin professor. Aussi la dérivation féminine professeuse ne serait-elle pas acceptable. Nous pouvons cependant objecter à ce raisonnement que ministre a été promptement féminisé, quoique auparavant des arguments du même genre aient été avancés ${ }^{10}$, et professeuse ou professeure ou professeur épicène au féminin ne peuvent nous surprendre. La productivité morphologique du français n'est pas si pauvre qu'on le prétend, et elle ne suffit pas à rendre compte du retard de la féminisation de professeur.

\section{Facteur sémantique}

D'après les auteurs de Femme, j'écris ton nom..., le genre grammatical du nom de métier doit s'accorder avec le sexe du référent s'il désigne un individu spécifique; mais il faut employer le genre masculin si le nom indique le métier ou le titre même. Cette opinion est illustrée par: «Dans cet hôpital, les fonctions de chirurgien (générique) sont occupées par une chirurgienne (spécifique)» (p. 39).

Nous supposons que cette contrainte sémantique devrait jouer un certain rôle dans le développement de la féminisation. Les noms de métiers dont nous traitons ici réfèrent par définition à une femme. Mais ils peuvent faire référence, suivant les contextes, soit à un individu, soit à la fonction exercée par cet individu, comme dans les exemples suivants :

Individu:

Pas de dragées ni de grains de riz pour le premier couple pacsé, mais peut-être la présence de la garde des Sceaux, \#Elisabeth\# Guigou. La ministre a, en effet, l'intention de bénir les premiers pacs, la semaine prochaine, dans un tribunal d'instance de Paris. (Libération, 1999)

L'AFMI s'est tournée vers \#Martine\# Anzani. Cet ancien juge d'instruction, très attaché à l'indépendance du judiciaire, préside aujourd'hui la chambre d'accusation de Paris. (Le Point, 1996)

9. Ce problème est discuté par exemple dans le chapitre «Les objections » de Femme, j'écris ton nom... (p. 27-30) : «Plusieurs objections reviennent régulièrement chez les opposants à la féminisation: l'homonymie, l'euphonie, la dévalorisation et la question du neutre » (p. 28). J. Rey-Debove écrit dans Le Monde (14 janvier 1998): "Le maintien du masculin (dans les exceptions comme ingénieur ou professeur) vient de la difficulté formelle de féminisation... »

10. On disait que la forme féminine de ministre est ministresse, qui indique l'épouse d'un ministre. 


\section{ITSUKO FUJIMURA}

\section{Fonction :}

Quand, au début de l'année, Alain-Dominique Perrin, le président de Cartier (ADP pour les intimes) lui offre le poste de directrice générale de Cartier France, \#Isabelle\# Guichot n'hésite que quelques heures. (Le Point, 1996)

Mercredi dernier se tenait, au dojo, l'assemblée générale extraordinaire du club, où les membres présents ont voté à l'unanimité pour la candidature au poste de président d'une femme dynamique et pleine d'idées, \#Elisabeth\# Derechniewski. (Le Télégramme, 2000)

Dans la figure 4, qui montre le changement de genre des noms de métiers avant et après 1998, fonction indique ceux qui sont précédés d'une expression telle que rôle de / poste de / fonction de / mandat de, tandis qu'individu indique ceux qui désignent un individu féminin évoqué au préalable dans un contexte antérieur ${ }^{11}$. On voit que la distinction sémantique entre la fonction et l'individu ne correspond pas nécessairement au genre grammatical, contrairement à la consigne de l'INaLF. Nous remarquons que les exemples du féminin désignant une fonction n'étaient pas si rares, même avant 1998, et ils continuent à augmenter, de même que ceux qui désignent un individu. Nous constatons seulement que l'usage du féminin s'est développé davantage dans le cas de référence à un individu plutôt que dans celui d'une fonction.

Figure 4: avant et après 1998 (1)

Fonction vs individu

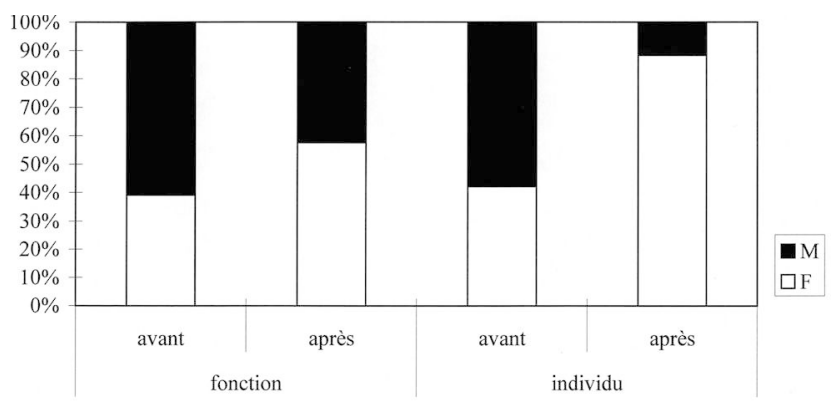

On pourrait conclure que cette distinction sémantique est l'un des facteurs de choix du genre grammatical. On ne peut pourtant pas croire que, dans le futur, l'usage du genre grammatical sera totalement conditionné par cette distinction, le féminin étant alors exclusivement utilisé pour un individu féminin et jamais pour une fonction occupée par cet individu.

11. Les noms apposés au nom propre ne sont pas pris en compte. 


\section{Facteur lexical}

Le facteur lexical est plus décisif que le précédent. La facilité de féminisation dépend notamment des noms, comme on le sait par expérience. La figure 5 montre les occurrences au masculin et au féminin de procureur(e), chercheur(se), député(e), avocat(e), conseiller(ère), directeur(rice) et président(e) avant et après 1998. La figure 6 offre ceux d'auteur, écrivain, maire, professeur, médecin, ministre, juge et secrétaire ${ }^{12}$.

On peut constater tout d'abord que la féminisation s'est développée après 1998 pour tous ces mots, même si l'on ne trouve que très peu de changements pour certains noms ; ministre, député(e) et juge ont notamment subi le changement le plus brutal; président(e), qui est féminisé aujourd'hui à $98 \%$, est le plus avancé dans la féminisation; procureur au féminin commence à apparaitre après 1998 sous forme de procureure, qui n'existait aucunement avant cette date.

Figure 5 : avant et après 1998 (2)

Les noms avec les deux formes stables

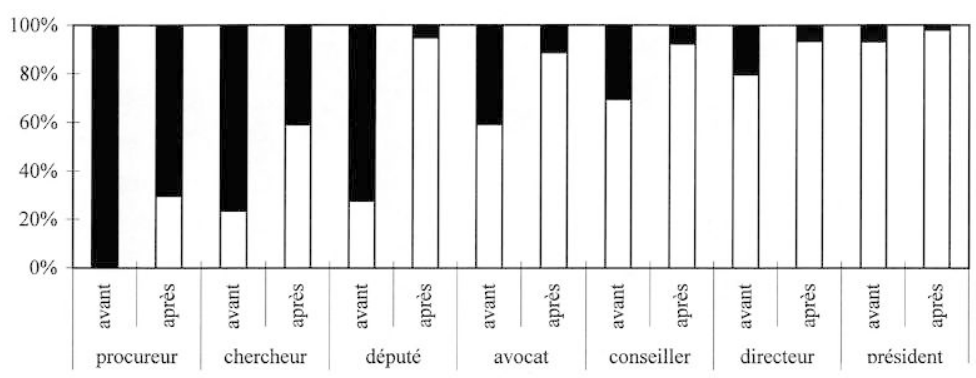

$\square \mathrm{F} \mathbf{M}$

Les noms qui ne se féminisent que très peu sont surtout auteur, écrivain, maire, médecin et professeur ${ }^{13}$. Par ailleurs, il faut souligner également le cas de chercheur(se): la fréquence de chercheur au féminin (chercheuse) n'atteint que $60 \%$ aujourd'hui, alors que conseiller(ère), directeur(rice) et avocat(e) se

12. Auteur, écrivain, maire, médecin et professeur sont appelés ici noms «constamment masculins » par commodité. Dans la figure 6, M et F indiquent les occurrences de ces noms avec une marque explicite de genre (ex.: un auteur). Les exemples sans marque explicite de genre (ex.: l'auteur) y sont catégorisés sous le symbole $M-$, parce que ces noms sans aucune marque précise de genre sont tout de même considérés en général comme masculins, d'après la forme du mot elle-même. Ministre, juge et secrétaire sont traités comme épicènes.

13. Voici des exemples rares de ces noms au féminin: "l'économiste \#Michèle\# Saint-Marc, ancienne auteur d'un “Que sais-je ?” sur le mark » (Libération, 1999); " La parole sera donnée à une écrivaine, \#Mireille\# Kuttel, auteur romand» (DNA, 2000); "La maire de Lille plaidait pour une "actualisation"» (Le Monde, 2001) ; " [...] pour remédier à la situation décrite par la médecin-chef de la Santé, \#Véronique\# Vasseur» (Le Monde, 2000). 
sont féminisés à plus de 90 \% après 1998. Le mot chercheur ne parait pas présenter de difficultés linguistiques en matière d’étymologie, ni de dérivation, ni d'homonymie dans son processus de féminisation. Pour expliquer ce décalage, il faut chercher la cause dans le monde extralinguistique plutôt que dans la structure de la langue.

Figure 6: avant et après 1998 (3) Les noms «constamment masculins » et les épicènes

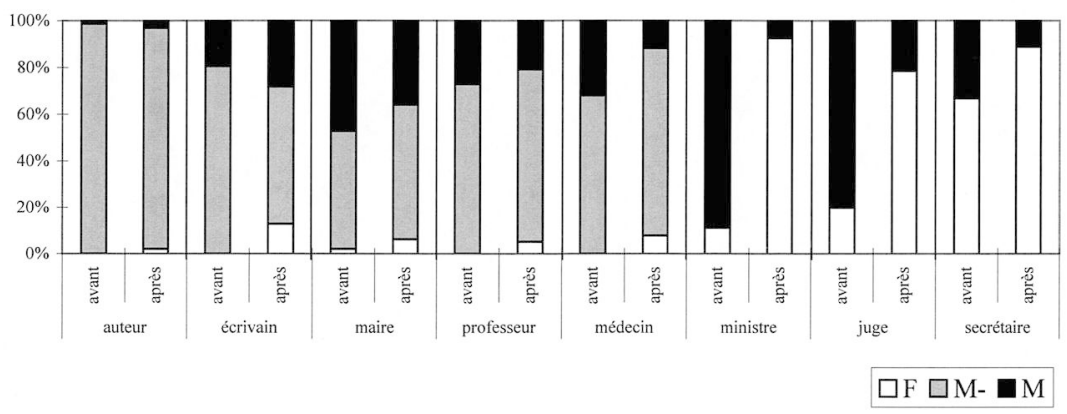

\section{Facteur sociolinguistique : types de métiers}

On fait souvent remarquer, implicitement ou explicitement, que la féminisation est conditionnée par les types de métiers plutôt que par les types de noms ${ }^{14}$. Le nom le plus fréquemment abordé pour illustrer ce phénomène est secrétaire. Une secrétaire désignait auparavant quelqu'un de sexe féminin qui travaillait pour le compte de son patron, alors que tous les secrétaires de haute situation tels que les secrétaires d'État, les secrétaires du bureau de l'Assemblée nationale, les secrétaires de partis politiques, étaient toujours exprimés avec le masculin, même si leur référent était une femme. Cet usage était contesté jusqu'ici surtout du point de vue de la justice sociale ou du «political correctness », parce que la différence de genre était en corrélation avec la hiérarchie sociale, qui pouvait être rapportée à la distinction entre les hommes et les femmes. On soulignait aussi que cette question concernait exclusivement les femmes, les hommes étant constamment désignés avec le genre masculin quel que soit leur statut social. Le domaine auquel les noms de métiers appartiennent est également un facteur important, comme le montrent les figures suivantes.

14. Les langues à genres grammaticaux n'ont pas toutes ce même caractère. En espagnol, par exemple, le genre grammatical des noms de métiers ne fonctionne que comme indicateur du sexe du référent, et non d'une sous-catégorie d'une lexie. 
La figure 7 relève les occurrences de directeur(rice), conseiller(ère) et président(e) avant et après $1998^{15}$. On voit que président du conseil régional, président du Parlement européen et conseiller municipal sont totalement féminisés après cette date. Parmi les "présidents", président du tribunal est le moins évolutif. Le cas le plus remarquable est la lenteur de féminisation de directeur de recherche. II nous semble irrationnel d'expliquer la différence de rythme d'évolution entre celui-ci et directeur général par leur position dans l'échelle sociale. II nous parait plus judicieux de souligner que directeur de recherche est un titre dans le domaine universitaire, de même que chercheur et professeur, tandis que directeur général est une fonction dans le domaine des affaires ou de l'administration ${ }^{16}$.

Figure 7 : avant et après 1998 (4) Types de métiers
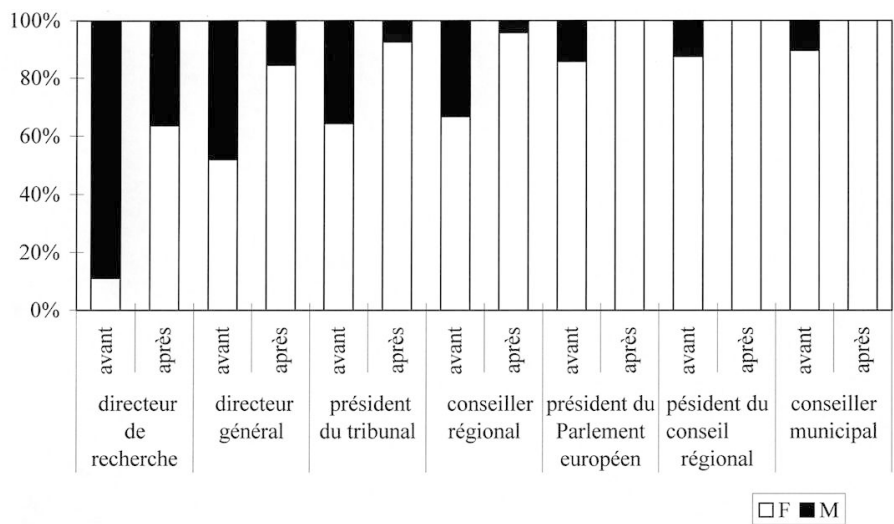

La figure 8, qui relève les noms des secrétaires de situation élevée avant et après 1998, montre clairement que leur genre grammatical a beaucoup changé depuis $1998^{17}$. Avant cette date, ces secrétaire étaient presque toujours au masculin. La seule exception remarquable concernait Nicole Notat, ancienne

15. Dans la figure 7, "président du tribunal» comprend les occurrences de président(e) de la chambre, de la cour. De même, " conseiller régional » inclut celles de conseiller(ère) général(e) et «président du conseil régional », celles de président(e) du conseil général. Sous la rubrique «président du Parlement européen » sont comprises les occurrences de président(e) de l'Assemblée du Conseil de l'Europe.

16. A.-M. Houdebine-Gravaud (1988) constate, d'après les enquêtes menées en 1983, 1984 et 1985, " une résistance plus grande à la féminisation » dans les domaines de l'armée, de la justice et de la médecine (p. 45).

17. Voici les fonctions détaillées de chaque personne :

Madeleine Albright: secrétaire d’État américain(e); Martine Auby: premier secrétaire de la fédération, secrétaire nationale du PS ; Anne-Marie Idrec, Nicole Catala, Florence Parly, Nicole Pery, Michelle Demessine : secrétaires d’État; Angela Merkel : secrétaire générale de la CDU; Hélène Carrère d’Encausse: secrétaire perpétuel de l'Académie; Nicole Notat: secrétaire général(e) de la CFDT. 
secrétaire générale de la CFDT, qui était désignée presque toujours avec la forme féminine (masculin : 1, féminin : 28). Après 1998, la situation s'est inversée. Les noms féminins constituent en effet depuis lors l'écrasante majorité. Madeleine Albright, ancienne secrétaire d’État américaine, désignée au masculin sans exception avant 1998, l'est aujourd'hui dans la plupart des cas avec le féminin (masculin: 19, féminin : 48). De même, toutes les femmes secrétaires d’État françaises sont désignées avec le féminin, contrairement à ce qu'on pouvait lire avant 1998. Cette forte tendance à la féminisation n'exclut pas cependant l'existence d'un contrexemple: Hélène Carrère d'Encausse, qui est devenue secrétaire perpétuel de l'Académie française en 1999. Cette nouvelle apparition de la forme masculine n'est pas sans rapport avec les difficultés de féminisation de certains noms, comme professeur, que nous avons examinés.

Figure 8 : avant et après 1998 (5) Secrétaires

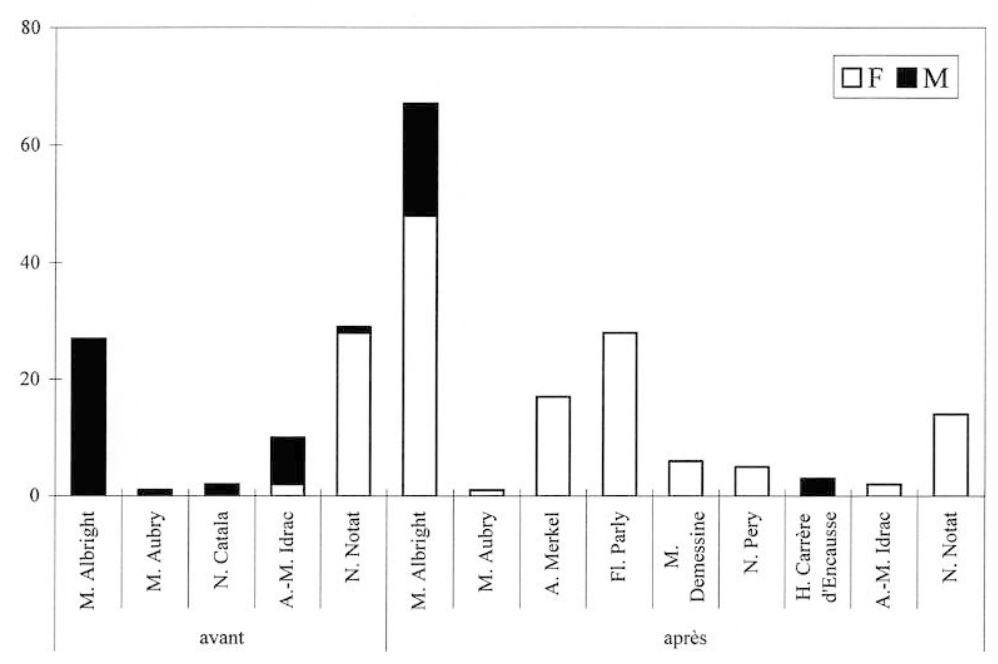

L’Académie a publié en 1984 une déclaration contre la féminisation signée par Georges Dumézil et Claude Lévi-Strauss, et la plupart des médias ont pris parti à l'époque pour cette position. Elle a ainsi réussi à entraver le mouvement du gouvernement, qui allait la réaliser. En 1998, l'Académie s'est une fois de plus opposée fermement à la féminisation, comme elle l'avait déjà fait dans les années quatre-vingt et continue de le faire encore aujourd'hui ${ }^{18}$. Ce qu'elle

18. Voir, par exemple, «La querelle du neutre», de M. Fumaroli, dans Le Monde (31 juillet 1998); "Féminisation: le rappel à l'ordre de l'Académie française », d'Anne Muratori-Philip, dans Le Figaro (23 mars 2002) ; plus récemment, toujours dans Le Figaro (9 décembre 2002) et sous la même signature, un article intitulé: "Hélène Carrère d’Encausse: “Le français, une cause nationale” ", où le journal évoque «l'idéologie responsable aussi d’une invention langagière désastreuse dont la féminisation des titres et fonctions». 
proclame constamment est le maintien du «bon français». Il n'est donc pas surprenant que les noms de métiers qui appartiennent au domaine des lettres ou de la recherche, en d'autres termes, les noms définissant les personnes qui sont étroitement liées au langage écrit et qui y sont attentives, présentent une attitude conservatrice vis-à-vis de cette évolution ${ }^{19}$. On peut conclure que ce serait un certain conservatisme linguistique qui aurait tendance à freiner la féminisation de mots comme professeur, écrivain, auteur, chercheur, directeur de recherche, secrétaire perpétuel de l'Académie. Il faut souligner que ce n'est pas seulement l'Académie en tant qu'institution particulière qui est à l'origine de ce conservatisme.

D'autre part, les institutions qui entreprenaient ce deuxième essai de féminisation étaient le gouvernement français, le président de la République et le Conseil de l'Europe ${ }^{20}$. Le but de ces dernières était de contribuer à résoudre des problèmes dans le monde réel en réformant la langue ${ }^{21}$. Les acteurs professionnels et institutionnels qui appartiennent aux sphères de la politique, de l'administration, de la justice et des affaires, qui s'intéressent surtout aux faits dans le monde extra-linguistique plutôt qu'aux faits linguistiques, acceptent facilement ce mouvement et l'encouragent. La féminisation s'y est rapidement accomplie, comme nous l'avons vu dans les cas de ministre, députée, juge, conseillère régionale, directrice générale, présidente du Parlement européen, secrétaire d'État. On pourrait voir là une forme de progression réaliste.

\section{Discussion}

Nous avons vu que dans la presse écrite française, la féminisation des noms de métiers a été accélérée en 1998 et continue à s'étendre actuellement. La forme manifestement masculine, avec un déterminant ou un adjectif masculin, a tendance à être évitée depuis 1998, même pour les noms dont la féminisation n'est pas encore très étendue. Le français pourrait être un jour une langue

19. E. Khaznadar (2000, p. 156) montre que, parmi les informatrices de tous les métiers au Québec, les enseignantes de français sont celles qui connaissent le moins les formes féminines officielles.

20. Voir «Recommandation 1229 de l'Assemblée parlementaire du Conseil de l'Europe relative à l'égalité des droits entre les hommes et les femmes " (1994): "L'Assemblée recommande au Comité des Ministres [...] de généraliser en français, dans le langage courant, des titres et dénominations de fonctions au féminin, et d'appliquer par exemple la circulaire française du 11 mars1986 relative à la féminisation des noms de métier, fonction, grade ou titre à ce sujet. »

21. La féminisation des noms de métiers atténuerait aussi le défaut linguistique du français. Dans l'exemple suivant, on peut se tromper sur l'identité du patron du HCR à cause du genre masculin de patron (Fujimura et Itoigawa, 2001) : «Malheureusement, le HCR est toujours utile. Et son patron actuel, Sadako Ogata, estime que le cinquantième anniversaire "n'est pas une occasion de réjouissance". Après une décennie à la tête du HCR, la Japonaise s'apprête à passer [...]» (Le Figaro, 16 décembre 2000). 
où le genre grammatical correspond exactement au sexe du référent. Ce qui serait une conséquence attendue non seulement du point de vue sociolinguistique, parce que l'on pourrait ainsi rendre le français sexuellement moins discriminatoire, mais aussi du point de vue purement linguistique, parce que la langue aurait un système de genre transparent.

Dans une langue à genres, le genre grammatical du nom correspond en principe au sexe du référent quand ce nom désigne un être humain. Si on est obligé de désigner par un nom masculin le poste d'une femme parce qu'il n'en existe pas d'équivalent féminin, cela posera des problèmes d'égalité entre les sexes parce que cette situation masque le fait que des femmes exercent dans ce domaine. Pour que les femmes soient visibles, il semble nécessaire de poursuivre la féminisation des noms de métiers et, dans de nombreux pays où la langue officielle est une langue à genres, on mène une telle politique.

On peut pourtant relever deux questions structurelles à propos de ce mouvement, l'une concernant la nature du système du genre grammatical dans toutes les langues à genres, l'autre, la caractéristique particulière du système du genre en français.

D'abord, la féminisation des noms de métiers ne saurait être une solution idéale, bien que favorable, pour que le français se transforme en langue non sexiste. Même si des noms féminins sont élaborés et utilisés pour tous les noms de métiers, tant que le système de genre persiste, les aspects inégalitaires de la langue ne disparaîtront pas. L'un d'eux est le masculin dit «générique », utilisé pour désigner la fonction seule, sans référence à une existence réelle ou potentielle; par exemple, un chirurgien dans une offre d'emploi. L'autre est le masculin pluriel qui désigne un groupe d'homme(s) et de femme(s), l'exemple typique étant ils incluant les deux sexes ${ }^{22}$. Cette caractéristique du français est exactement opposée à celle de l'anglais ou du japonais. Dans ces deux langues dépourvues de genre grammatical ${ }^{23}$, la réforme de la langue en vue de la réalisation de l'égalité des sexes consiste à faire disparaitre la différence formelle qui oblige à distinguer les deux sexes. Par exemple, en japonais, il a été décidé en 2002, dans le langage officiel et dans la presse écrite, de remplacer le mot kango-fu (infirmière: "soins-femme») par kangoshi («soins-maitre», sans distinction de genre), qui est un terme commun pour les infirmiers des deux sexes. L'enjeu du mouvement de féminisation en français est, de fait, le système du genre grammatical lui-même, qui ne nous parait pas favorable, paradoxalement, au but de ce mouvement.

22. Dans le langage écrit, on peut éviter ces usages par des moyens proposés, par exemple, dans le Guide pour la rédaction de textes non sexistes, 1988, ministère de l'Éducation du Québec.

23. En anglais, le genre persiste dans le système pronominal; par contre, en japonais, il n'y a aucun système de genre. Cela ne veut pas dire que cette langue ignorerait les questions relatives au genre. 
La deuxième question porte sur le retard de la féminisation du français parmi les langues à genres. Ce fait est mis en évidence par un surtitre du Monde (7 juillet 1998): "La France est l'un des derniers pays où la féminisation des titres fait débat. » La raison essentielle pour laquelle le français est beaucoup plus lent et soulève des difficultés sociolinguistiques bien plus grandes sur ce point que d'autres langues, notamment l'espagnol, est attribuable selon nous à la langue française elle-même plutôt qu'au climat de conservatisme linguistique en France, malgré l'avis de Suzanne Fleischman (1997) et de Marina Yaguello (1998).

Certes, au Québec, la féminisation s'est déclenchée plus tôt et a rencontré moins de résistance qu'en France. Mais cette différence n'est pas aussi radicale que celle entre le français et l'espagnol, que nous verrons ci-après. La situation au Québec s'explique par l'exigence plus forte de modernisation du français chez les Québécois, ainsi que par l'influence moins directe de l'Académie française ${ }^{24}$. En fait, le processus de féminisation est analogue dans ces deux nations. Au début, il y a un choc plus ou moins grand dans le public, quand quelqu'un commence à utiliser un mot féminisé. C'est par exemple ce qui s'est passé quand «Mme Lise Payette a été la première à exiger et à porter le titre de Madame la Ministre [en 1976], un titre qui a d'abord étonné, mais qui est entré très rapidement dans l'usage québécois» (M. de Villers, La Presse, 16 août 1997).

En revanche, le français contraste clairement avec l'espagno ${ }^{25}$. Dans cette langue, il est extrêmement facile d'accorder le genre d'un nom de métier au sexe de son référent. On fabrique un nom féminin en lui donnant un déterminant féminin sans changer sa forme, pour désigner une femme qui exerce cette profession. Par exemple, l'usage du nom féminisé la abogado (litt. «la avocat $»)^{26}$ est attesté depuis le début du $20^{\mathrm{e}}$ siècle, et la forme féminisée la abogada (litt. "la avocate») apparait un peu plus tard. Cette correspondance immédiate entre le sexe du référent et le genre grammatical du nom qui le désigne est comparable en français à celle du nombre, le nom étant mis au pluriel sans contrainte lorsque le référent est plural, alors que le genre grammatical en français est un fait prédéterminé dans le lexique : «Le nombre relève [...] de la quantification actuelle et est du domaine de la parole ; le genre [...] appartient [...] à la langue» (Bally, 1965, p. 80)

On pourrait expliquer cette différence par le fait que le genre en espagnol est plus transparent qu'en français. En espagnol, quand le nom désigne un inanimé, c'est le signifiant du nom qui compte dans la détermination du genre,

24. Nous partageons l'avis de J. Rey-Debove dans Le Monde (14 janvier 1998), qui condamne l'argument de l'Académie française comme non pertinent du point de vue linguistique. Voir aussi D. Leeman-Bouix, 1994.

25. Voir I. Fujimura \& M. Itoigawa, 2001.

26. C'est la féminisation dite minimale (voir A.-M. Houdebine-Gravaud, 1998, p. 22). 
dans la plupart des cas. On peut dire avec une forte probabilité que le nom est masculin s'il se termine en - $o$ et féminin s'il se termine en - $a^{27}$. S'il désigne une personne, de surcroit, il est en principe motivé par le sexe du référent. Même dans le cas contraire, comme persona qui est l'exemple représentatif de noncorrespondance entre le sexe et le genre, donc référentiellement non motivé, le genre de ce nom est évident par sa forme phonique en - $a$, donc phonétiquement motivé. Par contre, en français, comme le genre est soutenu dans de nombreux cas par la mémoire des locuteurs natifs pour chaque unité lexicale, on n'a aucun moyen de prévoir, de façon générale, le genre de personne sans connaitre la particularité de ce mot.

Le genre en français est un signe beaucoup plus «arbitraire » qu'en espagnol, dans l'acception saussurienne du terme. Et c'est pourquoi il peut acquérir un signifié. Nous partageons l'avis de Durrer (2002) qui affirme, suivant Bally: "Le sujet parlant, qu'on le veuille ou non, attribue [en français] une valeur sémantique au genre morphologique [...]. Ce n'est donc pas dans un contexte particulier que des connotations, des effets sont produits, mais ils sont inhérents aux substantifs. " La valeur sémantique attribuée au genre n'est pas, selon nous, nécessairement sexuée ; le genre grammatical fonctionne souvent comme outil distinctif des homonymes ou homophones en français: un/une livre, le /la tour, le/la voile, la/le politique. Les deux signifiés de secrétaire évoqués plus haut sont explicables de la sorte: le secrétaire n'avait pas auparavant la même valeur sémantique que la secrétaire, les deux unités lexicales signifiant des fonctions différentes. Si le remplacement de le professeur par la professeur(e) et de le ministre par la ministre tarde à être adopté en français, c'est parce que ce changement ne concerne pas simplement l'indication du sexe du référent, mais entraine aussi une modification du signifié du mot lorsque la nouvelle forme est mise au jour. Il faudra un certain temps pour que le nouveau mot soit accepté et diffusé parmi les francophones. Bref, en français, cette innovation équivaudrait à une création néologique, contrairement à l'espagnol où le genre des noms humains leur est attribué suivant la compréhension de l'univers référentiel, sans égard à la connaissance linguistique des noms.

Il nous semblerait parfait, à première vue, d'établir en grammaire française une règle selon laquelle seul le genre des noms humains correspond au sexe du référent, sans toucher au genre des noms non humains. La distinction entre ces deux catégories n'est cependant pas aussi nette qu'il y parait. Il existe de nombreux mots qui sont utilisés pour désigner les humains malgré leur origine non humaine, comme modèle, étoile, membre. La Belle et la Bête devrait-il devenir la Belle et le Bête?

27. $99,89 \%$ des noms en - 0 sont masculins, $96,6 \%$ des noms en - $a$ féminins et $89,35 \%$ des noms en -e masculins (U. K. Nissen, 2002, p. 252). 
Si le système du genre grammatical, opaque, irrégulier et complexe, est maintenu en français, c'est parce que l'usage de ce système est l'objet de contrôles normatifs par les autorités linguistiques: dictionnaires, livres de grammaire, enseignement, etc. Il va sans dire que l'Académie est le symbole de ces dernières. Mais les élites littéraires et universitaires jouent aussi un grand rôle à cet égard. L'irrégularité du système de genre est pour elles une source d'expressivité, parce que « [les irrégularités] mettent de la variété dans le discours» (Bally, 1965, p. 365).

Si le système de genre disparaissait du français, comme il l'a fait en anglais entre les $12^{\mathrm{e}}$ et $15^{\mathrm{e}}$ siècles, le caractère sexiste du français serait presque effacé, et de surcroit, l'apprentissage du français s'en trouverait facilité. Cependant, comme le genre grammatical remplit des fonctions, distinctive et expressive, cultivées tout au long de son histoire, la perte du système de genre apporterait à la langue française une détérioration importante.

Il n'y a pas de chemin idéal. Nous sommes face à un dilemme.

\section{Références}

BALLY Ch., 1965 [1932], Linguistique générale et linguistique française, $4^{\mathrm{e}}$ édition revue et corrigée, Berne, Francke.

BECQUER A., CERQUIGLINI B. et alii, 1999, Femme, j'écris ton nom... Guide d'aide à la féminisation des noms de métiers, titres, grades et fonctions, préface de L. Jospin, Paris, La Documentation française.

BOEL E., 1976, "Le genre des noms désignant les professions et les situations féminines en français moderne », Revue Romane, $\mathrm{n}^{0} 11$, p. 16-73.

DURRER S., 2002, "Les femmes et le langage selon Charles Bally: "des moments de décevante inadvertance" ? », Linguistique Online, $\mathrm{n}^{\circ} 11$.

FLEISCHMAN S., 1997, "The battle of feminism and bon usage: instituting nonsexist usage in French », The French Review, juin 1970, p. 834-844.

FUJIMURA I. \& ITOIGAWA M., 2001, «Féminisation des noms de métiers en français et en espagnol » [en japonais], Gengo Bunka Ronshu, $\mathrm{n}^{0}$ 23-1, p. 141-156.

FUJIMURA I., 2002, "Féminisation des noms de métiers en français dans le domaine de la politique: description diachronique » [en japonais], Gengo Bunka Ronshu, $\mathrm{n}^{\circ} 24-2$, p. 235-250.

HOUDEBINE-GRAVAUD A-M., 1988, "La féminisation des noms de métiers en français contemporain », Contrastes, p. 39-71.

— 1989, «Une aventure linguistique: la féminisation des noms de métiers, titres et fonctions en français contemporain », Terminologie et traduction, p. 91-145.

— 1987, «Le français au féminin », Paris, La Linguistique, ${ }^{0}$ 23-1, p. 13-34.

— éd., 1998, La féminisation des noms de métiers, Paris, L'Harmattan.

KHAZNADAR E., 2000, «Masculin et féminin dans la dénomination humaine: linguistique et politique. Aperçu de la pratique québécoise», Le français moderne, $n^{\circ}$ LXVIII-2, p. 141-170.

LEEMAN-BOUIX D., 1994, Les fautes de français existent-elles ?, Le Seuil. 


\section{ITSUKO FUJIMURA}

NISSEN U. K., 2002, "Gender in Spanish : tradition and innovation », Gender across Languages, vol. 2, M. Hellinger et H. Bußmann éd., p. 251-279.

YAGUELLO M., 1978, Les mots et les femmes, Paris, Payot.

— 1998, «Madame la ministre », Petits faits de la langue, Paris, Le Seuil, p. 118-139.

\section{Annexe}

\begin{tabular}{|c|c|c|c|}
\hline Périodique & Période & $\begin{array}{c}\text { Nombre } \\
\text { de mots } \\
\text { (en milliers) }\end{array}$ & Distributeur \\
\hline \multirow[t]{7}{*}{ Le Monde } & $01 / 02 / 1988$ & 3049 & $\begin{array}{l}\text { ELRA (European Language } \\
\text { Resources Association) }\end{array}$ \\
\hline & 01/02/1994 & 3603 & \\
\hline & 01/02/1997 & 4153 & \multirow[t]{2}{*}{ CEDROM-SNi } \\
\hline & $01 / 02 / 1998$ & 4264 & \\
\hline & $01 / 02 / 2000$ & 4324 & ELRA \\
\hline & $01 / 02 / 2001$ & 5171 & http://www.lemonde.fr/ \\
\hline & total & 24654 & \\
\hline \multirow{3}{*}{$\begin{array}{l}\text { Libération } \\
\text { (Libé) }\end{array}$} & 01/02/1995 & 3512 & CEDROM-SNi \\
\hline & $11 / 12 / 1999$ & 2403 & \\
\hline & total & 5915 & \\
\hline \multirow{3}{*}{$\begin{array}{l}\text { Le Télégramme } \\
\text { (Télé) }\end{array}$} & $05 / 1996$ & 4842 & http://www.letelegramme.com/ \\
\hline & $10 / 2000$ & 8453 & \\
\hline & total & 13295 & \\
\hline \multirow{3}{*}{$\begin{array}{l}\text { Dernières Nouvelles } \\
\text { d'Alsace } \\
\text { (DNA) }\end{array}$} & 01/02/1996 & 2549 & http://www.dna.fr/dna/ \\
\hline & $09 / 10 / 2000$ & 4054 & \\
\hline & total & 6603 & \\
\hline Le Point & 1996 & 3180 & CEDROM-SNi \\
\hline \multirow[t]{2}{*}{ (Point) } & 2000 & 3738 & \\
\hline & total & 6918 & \\
\hline \multicolumn{2}{|l|}{ TOTAL } & 57295 & \\
\hline
\end{tabular}

